\title{
Activity of Relatively Close Binaries
}

\author{
Carolus J. Schrijver ${ }^{1}$, Cornelis Zwaan ${ }^{2}$ \\ ${ }^{1}$ Space Science Department of ESA, ESTEC, Mail Box 299, 2200 AG \\ Noordwijk, The Netherlands \\ ${ }^{2}$ Astronomical Institute, University of Utrecht, Mail Box 80 000, $3508 \mathrm{TA}$ \\ Utrecht, The Netherlands
}

\begin{abstract}
Whereas the rotation-activity relationship for single cool stars can be described in terms of fundamental stellar properties, the activity in relatively close binaries is enhanced with respect to that of single stars by an amount which depends strongly on the properties of the companion. We consider mechanisms which could cause an excess in activity of binaries, and conclude that it is likely that the presence of a companion affects the interior structure of the stars in such a way that either the efficiency of the dynamo or of the atmospheric heating is enhanced.
\end{abstract}

\section{Introduction}

Magnetic activity, apparently ubiquitous in stars with convective envelopes immediately beneath the surface, is intimately connected with stellar rotation. Those cool stars which are either single or a component of wide binary systems define colour-dependent relationships between activity and rotation rate (e.g. Walter and Bowyer, 1981; Rutten, 1984; Schrijver et al., 1984, Pallavicini et al., 1985). Figure 1 , however, shows that some stars are much brighter in chromospheric and coronal emissions than would be expected from the rotation-activity relationships defined by single stars (e.g. Rutten, 1987). Basri et al. (1985) had also noted that subgiants in synchronized binary systems were more active than single stars with the same rotation period. The "overactivity" in soft X-rays can be as large as an order of magnitude for rotation periods below about 30 days, and even larger at longer periods. These "overactive" stars are generally components of relatively close binary systems, or are otherwise unusual stars such as pre-main sequence stars, contact binaries or merged binaries. We perform a search for the parameter(s) describing the overactivity. 


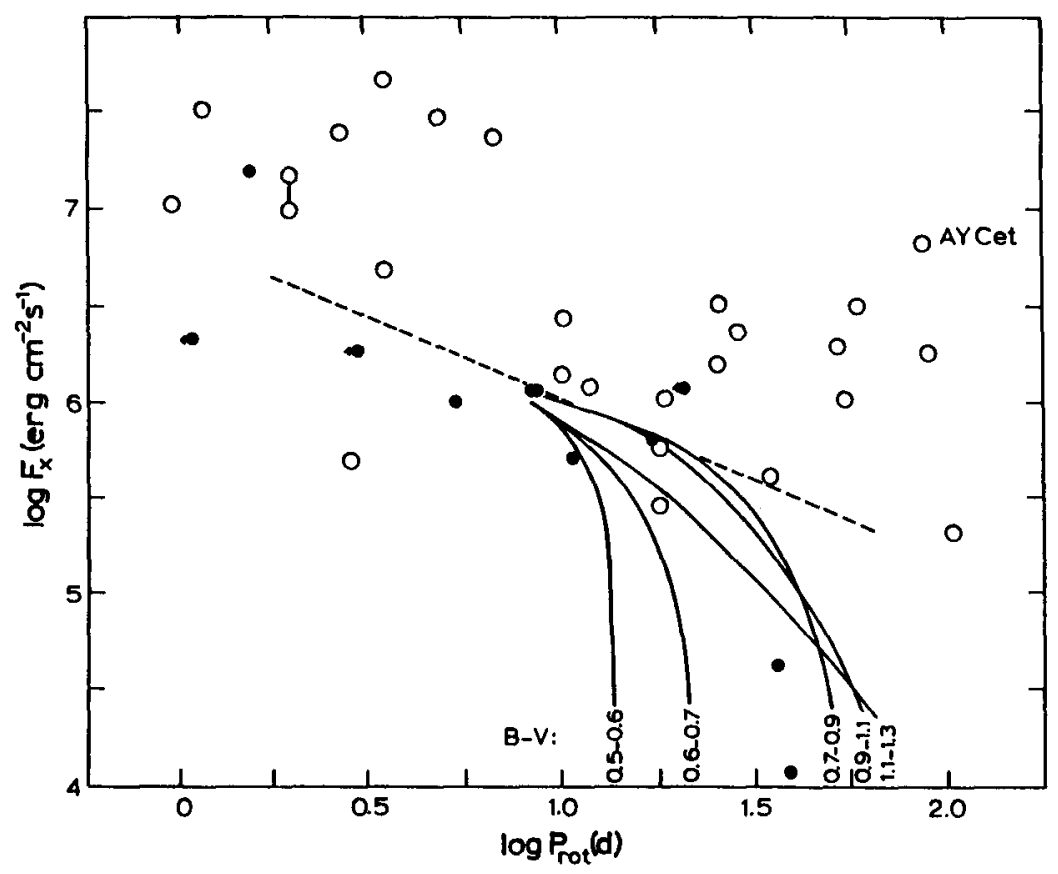

Fig. 1. Rotation-activity diagram: plotted are the surface flux density in soft X-rays, $F_{X}$, versus rotation period $P_{\text {rot }}$. The small dots show the location of single stars. The relatively close binaries are shown as circles. The curves show the colour-dependent rotation-activity relationships derived by Rutten (1987) using a sample of about 300 single stars and wide binaries. The dashed line shows the approximation of Eq. (2).

\section{Selection of data}

We selected stars from the lists of Schrijver et al. (1984) and of Strassmeier et al. (1990) for which sufficient information was available to derive the basic parameters of at least the primary (such as rotation rate, mass, radius, effective temperature, etc.), of the orbit (period, separation, inclination, and eccentricity), and of the activity level with at least the soft X-ray flux known. Our data set includes 21 binary systems for which the properties of the individual components and of the orbits are reasonably well known, and four additional systems with less certain parameters (see Schrijver and Zwaan, 1990, and Fig. 2). Eleven single stars are used for a detailed comparison, while the mean rotation-activity relations derived by Rutten (1987), based on a large sample of stars, are used as a reference. Activity is measured by coronal soft X-ray fluxes, $F_{X}$, observed with the the HEAO-2 EINSTEIN satellite, UV fluxes observed with the International Ultraviolet Explorer, and $\mathrm{Ca}$ II $\mathrm{H}+\mathrm{K}$ fluxes observed with the Mt. Wilson HK spectrophotometer (see Schrijver and Zwaan, 1990, for more details).

Optical data show that if the two binary components are not of comparable size, the bulk of the atmospheric radiative losses appears to come from the cool primary (unless the warmer secondary is a rapidly spinning star like in Capella). 
Hence the fluxes are usually assigned to the primary, except in cases where evidence exists that both components are active (see Schrijver and Zwaan, 1990).

\section{Rotation and activity}

A striking feature of the the radiative losses from the outer atmospheres of cool stars is that they are highly correlated among themselves, defining simple powerlaw relationships (e.g. Schrijver, 1987). Moreover, the basic stellar parameters do not enter into these relationships, provided one uses the radiative losses in excess of the so-called basal flux (which is probably unrelated with magnetic activity). Because of this property, Oranje et al. (1982), Schrijver (1983), Zwaan (1983), Oranje (1986) and Basri (1987) have suggested the existence of a single "activity parameter." This activity parameter is a function of several basic stellar parameters with the rotation rate playing a dominant role. Given the activity parameter, all radiative losses from chromosphere, transition region, and corona can be calculated without further knowledge of the star in question. The relatively close binaries generally follow the trend defined by the much larger sample of single stars and wide binaries. Although this suggests comparable differential emission measure distributions for single stars and for binaries throughout most of the atmospheres, we caution that it is not clear whether the coronal atmospheric structure is the same because too little is known about the coronal structure from the available broad-band measurements. Because of the interdependence of the diagnostics of activity, we concentrate on the soft $\mathrm{X}$-ray fluxes in this analysis of overactivity.

A principal component analysis performed on the logarithmic values of the soft X-ray flux, $F_{X}$, the orbital period, $P_{o r b}$, the binary separation, $d$, the radii of the hot and cool components, $R_{h}$ and $R_{c}$, and the corresponding masses, $M_{h}$ and $M_{c}$, suggests that the data span a two-dimensional subspace (covering $87 \%$ of the variance). We subsequently derived a two-parameter description of the activity level for the binaries in our sample:

$$
F_{X}=610^{7} \frac{R_{h}}{P_{o r b}^{1.3}}
$$

$F_{X}$ in erg $\mathrm{cm}^{-2} \mathrm{~s}^{-1}, R_{h}$ in solar radii, $P_{\text {orb }}$ in days. Eq. (1) describes the data with a $1 \sigma$ deviation of a factor of 1.8 (Fig. 2), which is quite acceptable given the uncertainties in the stellar and orbital parameters and the uncertainties in and the expected variability of the soft X-ray fluxes. It is clear from Eq. (1) that some property of the companion is indispensible in this relationship. We cannot distinguish whether the rotation or orbital period should be used, although the fit is somewhat better if $P_{\text {orb }}$ is used.

The expected emission for a single star of a given period with $P_{\text {rot }} \leq 30$ days (Rutten, 1984) can be approximated by:

$$
F_{X, 0}=710^{6} P_{\text {rot }}^{-0.8} \text {. }
$$




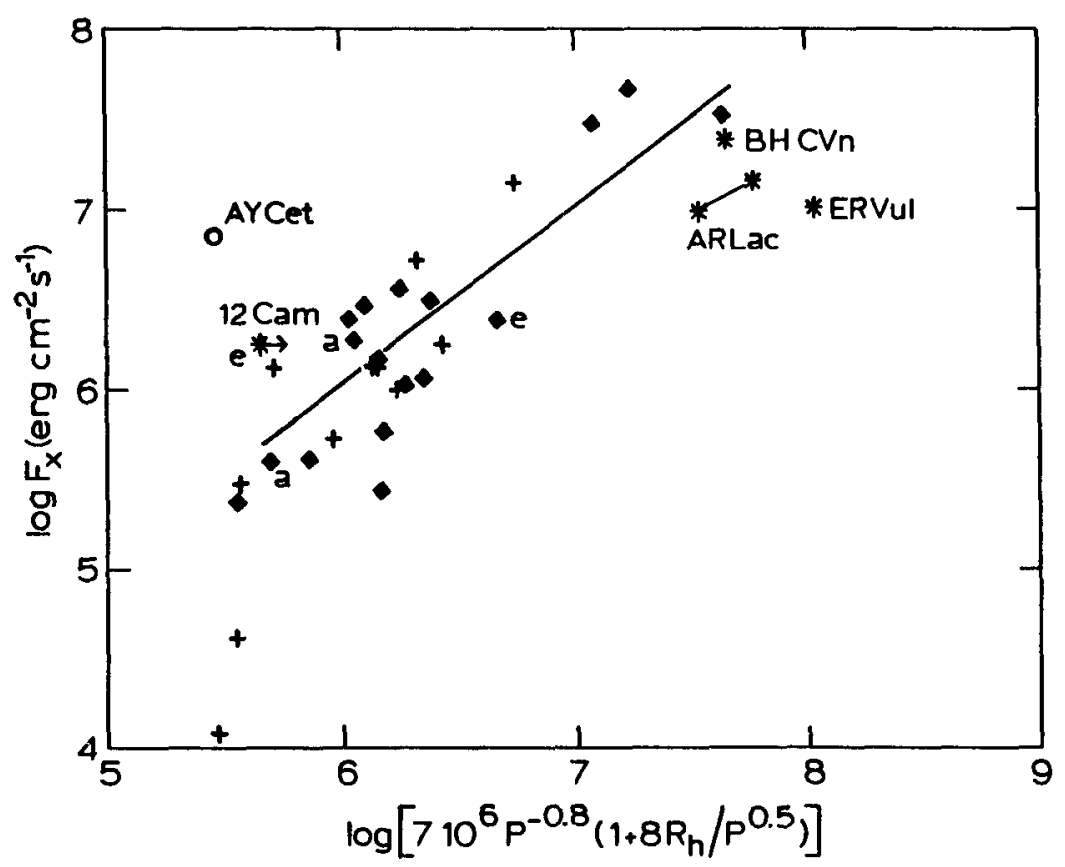

Fig. 2. Best-fit power-law parameterization of the rotation-activity relationship for single stars and for close binaries. The soft $\mathrm{X}$-ray flux density is plotted vs. the best-fit value computed using Eq. (4). Symbols: $\diamond$ close binaries, * short-period systems or DA companion, + single stars.

Single stars and binaries can be combined in the approximate relationship (Fig. 2):

$$
F_{X}=7.10^{6} P_{o r b}^{-0.8}\left(1+8 * \frac{R_{h}}{\sqrt{P}}\right)=F_{X, 0}\left(1+8 * \frac{R_{h}}{\sqrt{P}}\right)
$$

(units as in Eq. (1)). In this relationship the activity in close binaries is expressed as a true "overactivity", i.e. an enhancement compared to the expected level of activity in a single star of the same rotation period.

Although Eqs. (1) and (3) use the radius of the companion to describe overactivity, we caution that there are strong dependences between the fundamental parameters of the stars in our sample, which could transform the relationship into a seemingly different representation of the same basic dependence. For the binary components, for instance, we find ( $M, d$, and radii $R$ in solar units, periods $P$ in days): $P_{o r b}=0.12 * d^{1.34}$, (because of Kepler's law; correlation coefficient $C=0.983$ for logarithmic values) $d=5.5 * R_{c}^{0.93}(C=0.74), M_{c}=0.55 * R_{c}^{0.56}(C=0.92)$ and $M_{h}=1.1 * R_{h}^{0.33}(C=0.79)$.

If the secondary star can influence the activity of the primary, the primary may also affect the secondary. In close binaries with components with comparable masses and radii this appears to be the case. For binaries in which the secondaries are much smaller than the primaries, the enhanced activity of the secondary may not be detectable, or the activity enhancement may not occur in the same way. 
There is some evidence that the activity (including the overactivity) in a few binary systems (ER Vul, BH CVn and AR Lac, shown by asterisks in the upperlefthand corner of Fig. 2) is somewhat reduced compared to that in systems with longer periods. This "saturation" is a well-known property of W UMa systems (Vilhu and Rucinski, 1983, but see Zeilik, these Proceedings). These systems have therefore been excluded from the fitting procedure described above.

We have shown that the level of activity in relatively close binaries is determined not only by the rotation or revolution period, but also by basic properties of the companion. In the interpretation of the data it not clear whether the soft $\mathrm{X}$-ray flux should be interpreted as an additional activity (implicit in the representation chosen for Eq. (1)), more or less unconnected with the ordinary magnetic activity, or as an enhancement of the expected level of magnetic activity given the rotation rate of the primary (as in $\mathrm{Eq}$. (3)).

Schrijver and Zwaan (1990) consider several possible mechanisms which could cause overactivity in close binaries. They conclude that it is unlikely that the energy required for the overactivity is generated near the hotter companion, and then transported to the cool primary, thus eliminating accretion processes occurring near the hotter component, and magnetic coupling of the stars by the interstellar magnetic field. Instead, the presence of a companion may affect the interior structure of the cool primary in such a way that the efficiency of the dynamo (possibly through changes in the differential rotation) or of the atmospheric heating (by a modification of some characteristic of the top of the convection zone) is enhanced.

\section{References}

Basri, G.: 1987, Astrophys. J. 316, 377

Basri, G., Laurent, R., Walter, F.M.: 1985, Astrophys. J. 298, 761

Oranje, B.J., Zwaan, C., Middelkoop, F.: 1982, Astron. Astrophys. 110, 30

Oranje, B.J.: 1986, Astron. Astrophys. 154, 185

Pallavicini, R., Golub, L., Rosner, R., Vaiana, G.S., in Second Cambridge Workshop on Cool Stars, Stellar Systems, and the Sun, eds. M.S. Giampapa and L. Golub, SAO Spec. Rep. 392, Vol. II, p. 77

Vilhu, O., Rucinski, S.M.: 1983, Astron. Astrophys. 127, 5

Rutten, R.G.M.: 1987, Astron. Astrophys. 177, 131

Rutten, R.G.M., Schrijver, C.J., Lemmens, A.F.P., Zwaan, C.:1990, in prep. for Astron. Astrophys.

Schrijver, C.J.: 1983, Astron. Astrophys. 127, 289

Schrijver, C.J.: 1987, Astron. Astrophys. 172, 111

Schrijver, C.J., Mewe, R., Walter, F.M.: 1984, Astron. Astrophys. 138, 258

Schrijver, C.J., Zwaan, C.: 1990, submitted to Astron. Astrophys.

Strassmeier, K.G., Hall, D.S., Boyd, L.J., Genet, R.M.: 1989, Astrophys. J. Suppl. 69, 141

Vilhu, O., Rucinski, S.M.: 1983, Astron. Astrophys. 127, 5

Walter, F.M., Bowyer, S.: 1981, Astrophys. J. 245, 671

Zwaan, C.: 1983, in Solar and Stellar Magnetic Fields: Origins and Coronal Effects, ed. J.O. Stenflo, Reidel, p. 85 